\title{
Magnetophonon oscillations of thermoelectric power and combined resonance in two-subband electron systems
}

\author{
A. D. Levin, ${ }^{1}$ G. M. Gusev, ${ }^{1}$ O. E. Raichev, ${ }^{2}$ and A. K. Bakarov ${ }^{3,4}$ \\ ${ }^{1}$ Instituto de Física da Universidade de São Paulo, 135960-170, São Paulo, SP, Brazil \\ ${ }^{2}$ Institute of Semiconductor Physics, NAS of Ukraine, Prospekt Nauki 41, 03028 Kyiv, Ukraine \\ ${ }^{3}$ Institute of Semiconductor Physics, Novosibirsk 630090, Russia and \\ ${ }^{4}$ Novosibirsk State University, Novosibirsk 630090, Russia
}

(Dated: October 3, 2018)

\begin{abstract}
By measuring the thermoelectric effect in high-mobility quantum wells with two occupied subbands in perpendicular magnetic field, we detect magnetophonon oscillations due to interaction of electrons with acoustic phonons. These oscillations contain specific features identified as combined resonances caused by intersubband phonon-assisted transitions of electrons in the presence of Landau quantization. The quantum theory of phonon-drag magnetothermoelectric effect, generalized to the case of multi-subband occupation, describes our experimental findings.

PACS numbers: 73.43.Qt, 73.50.Lw, 73.63.Hs
\end{abstract}

\section{INTRODUCTION}

It has been recently established that magnetotransport coefficients of two-dimensional (2D) high-mobility electron gas in quantum wells (QWs) demonstrate magnetophonon oscillations (MPO) due to interaction of electrons with acoustic phonons [1-16]. These oscillations are caused by a combined effect of Landau quantization in the perpendicular magnetic field $B$ and sensitivity of electron-phonon scattering probability to wavenumbers of acoustic phonon modes dictated by the kinematics of scattering near the Fermi surface. The backscattering processes, when the phonon wavenumber $Q$ is close to the Fermi circle diameter $2 k_{F}$, have a maximum probability. On the other hand, the Landau quantization implies that the highest scattering probability is realized when phonon frequency is a multiple of the cyclotron frequency $\omega_{c}=|e| B / m c$. Since the acoustic phonon frequency is given by a linear relation $\omega_{\lambda \mathbf{Q}}=s_{\lambda} Q$, where $s_{\lambda}$ is the sound velocity of the mode $\lambda$, the transport is enhanced under the magnetophonon resonance conditions $2 k_{F} s_{\lambda}=n \omega_{c}$, where $n$ is an integer. As the magnetic field changes, different Landau levels enter the resonance, and the $1 / B$-periodic oscillating picture appears. These oscillations are not sensitive to the position of the Fermi level with respect to Landau levels, so they are much more robust to increasing temperature $T$ than the Shubnikov-de Haas oscillations. Moreover, the amplitude of the oscillations increases with $T$ in the Bloch-Gruneisen region $T<2 p_{F} s_{\lambda}$ due to increase in the number of phonons contributing to the electron-phonon collisions.

The acoustic MPO of electrical resistance, also known as phonon-induced resistance oscillations, have been observed in numerous experiments $[1,3,4,6,7,9,13]$. They are well seen under the conditions when phonons play a significant role in relaxation of electron momentum, for example, in QWs of very high quality [6] where electronimpurity scattering is minimized, or at elevated temperatures [3]. Measurements of thermoelectric power (thermopower) in GaAs QWs also show acoustic MPO
$[2,16]$, because the thermoelectric phenomena [17] in GaAs quantum wells are caused mostly by the phonon drag mechanism $[17,18]$. The resistance is determined by both electron-phonon and electron-impurity scattering, the latter prevails at low temperatures. In contrast, the longitudinal (Seebeck) thermopower due to phonon drag is determined solely by the electron-phonon scattering, though the electron-impurity scattering remains important for shaping the density of states of electrons in magnetic field. Therefore, the studies of magnetothermopower is a more direct way for investigation of acoustic MPO as compared to the studies of magnetoresistance. However, the reported observations $[2,16]$ of these oscillations in thermopower are very sparse and no detailed comparison of experimental data to theoretical calculations has been done so far.

In QWs with two or more occupied 2D subbands, there exists another type of quantum oscillations due to scattering of electrons between the subbands. These magneto-intersubband oscillations (MISO) [19-34] observed in the resistance measurements are governed by the magneto-intersubband resonance, when the difference in subband energies is a multiple of the cyclotron energy. In these conditions, the impurity-assisted elastic scattering of electrons between the subbands becomes significant and enhances the total scattering probability. In twosubband system with subband separation $\Delta$, the magnetoresistance shows $1 / B$-periodic MISO with maxima at $\Delta=n \hbar \omega_{c}$. Similar to magnetophonon oscillations, the MISO are robust to increasing temperature, they were detected at $T$ up to $40 \mathrm{~K}$ [24]. The MISO with high amplitudes and large period are commonly observed in magnetoresistance of double layer structures (such as double QWs [24] or single wide QWs studied in this paper, see Fig. 1), where subband separation is small and intersubband scattering is strong.

Recently, measurements of magnetoresistance of a twosubband electron system in a single QW with high electron density have revealed an interesting phenomenon, which can be viewed as the interference of acoustic MPO 


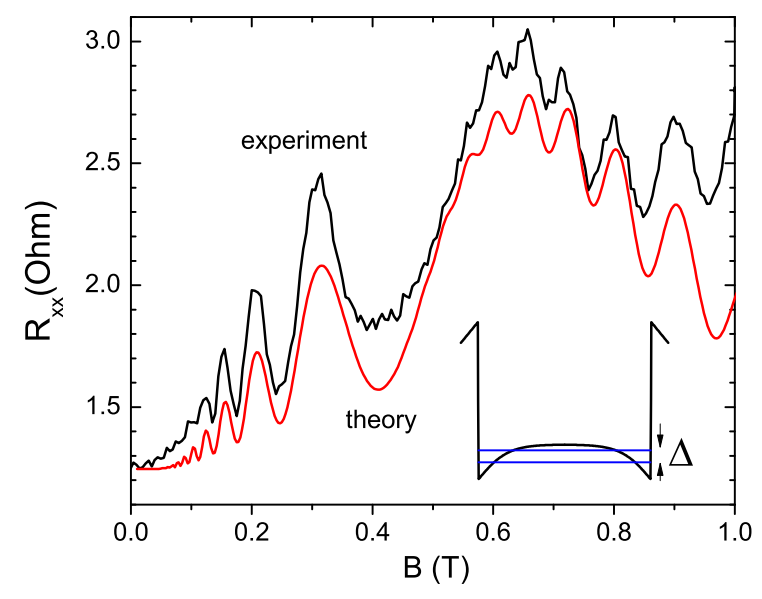

FIG. 1: (Color online) Magneto-intersubband oscillations of resistance in the wide $\mathrm{QW}$ samples at $T=4.2 \mathrm{~K}$. A comparison of the measured magnetoresistance with the calculated one allows us to determine subband separation $\Delta=1.07 \mathrm{meV}$ by the oscillation frequency and to estimate the quantum lifetime of electrons by the amplitude of the oscillations.

with MISO [9]. In terms of microscopic quantum processes, such a phenomenon exists because of phononassisted intersubband scattering of electrons. Under Landau quantization, this scattering leads to magnetoresistance oscillations whose periodicity is governed by commensurability of cyclotron energy with combined energies defined as a sum and a difference of the subband separation energy $\Delta$ and the characteristic phonon energy $\hbar\left(k_{1}+k_{2}\right) s_{\lambda}$, where $k_{1}$ and $k_{2}$ are the Fermi wavenumbers for subbands 1 and 2 . Thus, the combined resonance conditions are $[9,11]$

$$
2 k_{F} s_{\lambda} \pm \Delta / \hbar=n \omega_{c}
$$

where $k_{F}=\left(k_{1}+k_{2}\right) / 2$. The changes in magnetoresistance associated with the interference in Ref. 9 were definitely resolved near the main magnetophonon resonance around $B=1 \mathrm{~T}$. In a wide region of $B$, the behavior of the magnetoresistance was governed rather by a superposition of MPO and MISO, due to phonon-assisted intrasubband and impurity-assisted intersubband contributions to transport, respectively.

The interference of MPO with MISO is an important phenomenon because it is a unique manifestation of interference of two distinct types of quantum magnetooscillations in quasi-equilibrium macroscopic transport coefficients [35]. However, Ref. 9 still remains a single report of the observation of the combined resonances (1) in magnetotransport. In this paper, we propose to employ the measurements of phonon-drag thermopower as a more convenient method for observation of the combined resonances, compared to the resistance measurements. The longitudinal thermopower is determined by electron-phonon scattering and, therefore, does not show up the intersubband resonances due to elastic electronimpurity scattering. This property facilitates detection of the combined resonances caused by the intersubband phonon-assisted scattering. It is worth noticing that previous measurements of the thermopower in two-subband electron systems [36-38] were concentrated on different subjects and did not reveal either the MPO or the combined resonances.

Below we report both experimental and theoretical studies of the magnetothermopower of a two-subband electron system in a wide $(45 \mathrm{~nm})$ GaAs QW. Because of charge redistribution, a wide QW forms a bilayer (see the inset in Fig. 5), where two wells near the interfaces are separated by an electrostatic potential barrier, and two subbands appear as a result of tunnel hybridization of $2 \mathrm{D}$ electron states. The magnetoresistance of our system shows pronounced MISO corresponding to $\Delta=1.07$ $\mathrm{meV}$. This value of intersubband separation is close to that $(0.95 \mathrm{meV})$ obtained from a self-consistent calculation of subband spectrum and wave functions. While measuring the magnetothermopower, we naturally do not see the MISO, but observe a considerable change of magnetooscillation picture compared to that in singlesubband QWs. Theoretical calculations satisfactory describe our findings, thereby confirming the importance of phonon-assisted intersubband scattering in phonon-drag magnetothermoelectric effect.

The paper is organized as follows. Section II describes experimental part and the results. The details of the theoretical analysis are given in Sec. III. A comparison of the theory with the experiment, discussion of the results, and concluding remarks are presented in Sec. IV.

\section{EXPERIMENT}

We have studied both narrow $(w=14 \mathrm{~nm})$ and wide $(w=45 \mathrm{~nm})$ GaAs QWs with electron density $n_{s}=6.4 \times 10^{11} \mathrm{~cm}^{-2}$ and mobility $1.9 \times 10^{6} \mathrm{~cm}^{2} / \mathrm{V}$ s. The samples were made in a modified van der Pauw geometry, with an electrically powered heater placed at the side of the sample, several millimeters away from the $2 \mathrm{D}$ layer. The $2 \mathrm{D}$ electron gas occupies a circular central part (diameter $1 \mathrm{~mm}$ ) and four long (length $5 \mathrm{~mm}$, width $0.1 \mathrm{~mm}$ ) arms ending with the voltage probes ( se figure 2 ). The thermoinduced voltage $V$ was measured by a lock-in method at the frequency of $2 f_{0}=54 \mathrm{~Hz}$. The measurements have been carried out at $T=4.2 \mathrm{~K}$. We find the electron temperature near the heater and heat sink by the 2-probe measurements, exploiting the amplitude of the Shubnikov-de Haas oscillation. The difference in the electron temperature between hot and cold sides is found $\Delta T \simeq 0.1-0.2 \mathrm{~K}$ at the lattice temperature $T=4.2 \mathrm{~K}$. Several devices with narrow and wide QWs from two wafers have been studied. Figure 2 illustrates magnetic-field dependence of the thermoinduced voltage for narrow and wide QWs. The voltage increases nearly 
linearly with heater power and is almost symmetric with respect to the sign of the magnetic field, which proves that we measure the longitudinal (Seebeck) thermoelectric effect. In both cases, we see MPO confirming that the contribution to the thermoelectric effect comes from the phonon drag mechanism.
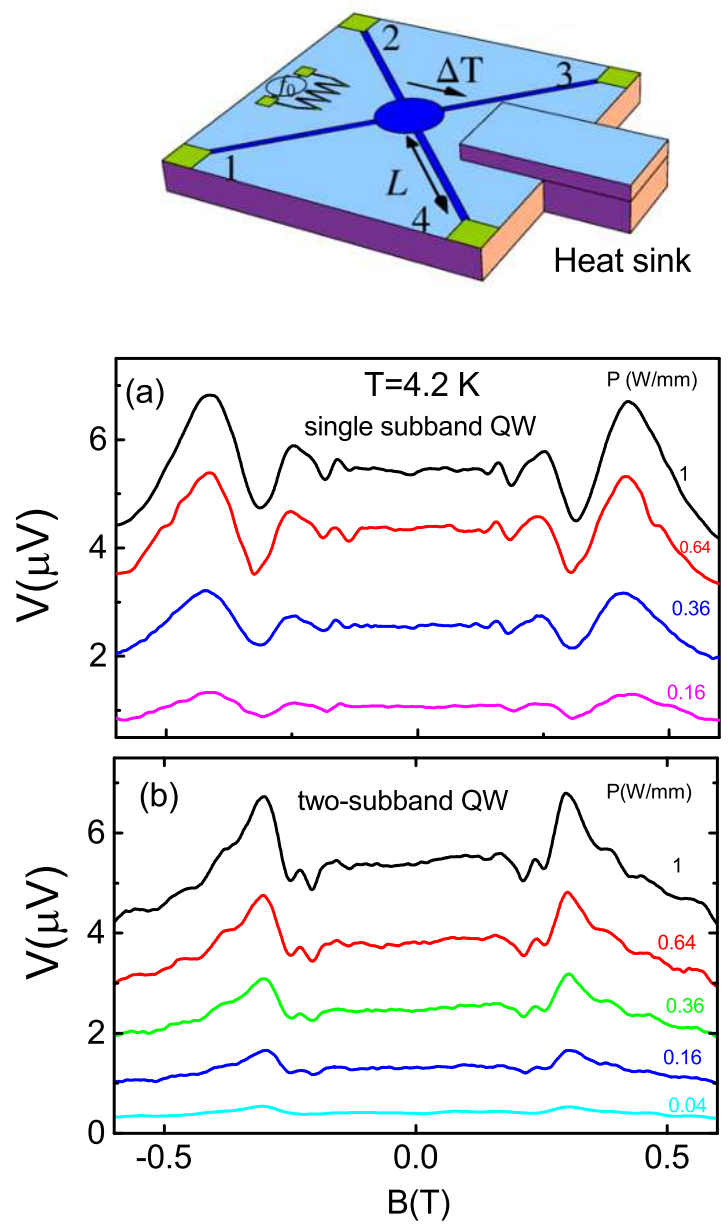

FIG. 2: (Color online) Sample geometry and magnetic-field dependence of the thermoinduced voltage for single-subband (a) and two-subband (b) QWs for different heater powers.

In Fig. 3 we plot the first derivative of the thermopower signal with respect to $\mathrm{B}$, allowing to the position of the minima and maxima for narrow and wide QWs to be compared. The oscillation position in both systems is coincident only in low magnetic field. At higher field the position of peak in single QW follows to $1 / B$ period, while two-subband system exhibits several additional oscillations.

Figures 4 and 5 present more detailed plots of the normalized thermoinduced voltage in single and twosubband QWs for a chosen heater power together with theoretical calculations. In the single-subband QW the MPO resembles the ones obtained in the previous experiment [2]. In the two-subband QW we observe a more complicated oscillating picture showing several weaker

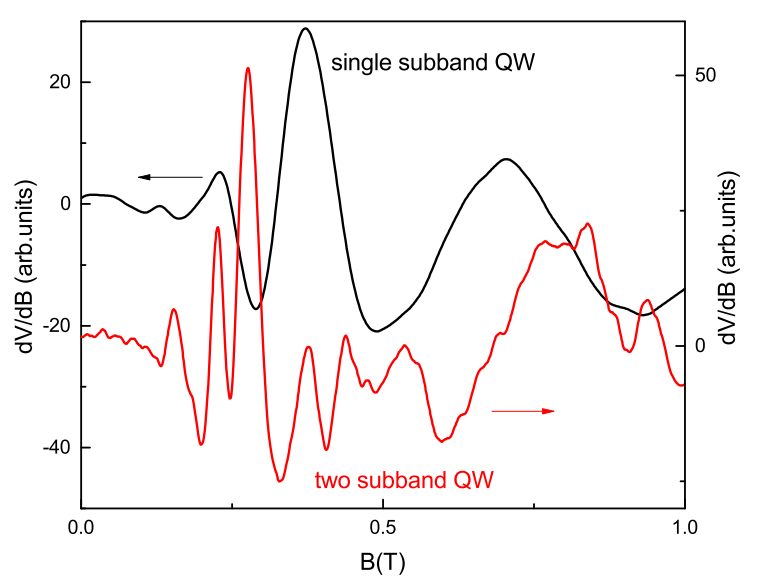

FIG. 3: (Color online) First derivative of the thermoinduced voltage with respect to B for single and two-subband QWs.

resonances and an unexpected growth of the thermoinduced voltage with magnetic field at $B>0.7 \mathrm{~T}$. The theoretical analysis given below allows us to identify these specific for two-subband QWs features and explain them as a result of intersubband phonon-assisted transitions.

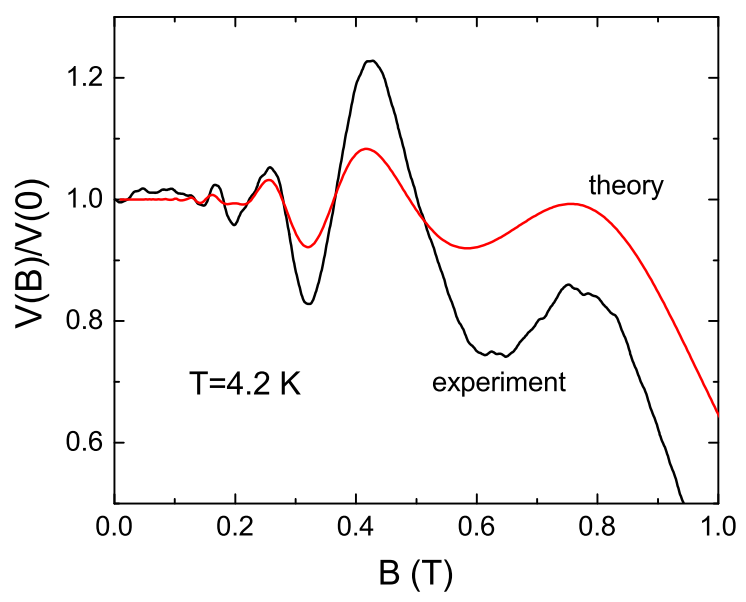

FIG. 4: (Color online) Magnetic-field dependence of the normalized thermoinduced voltage for single-subband QW.

\section{THEORY}

For multi-subband systems, the kinetic theory describing 2D electrons interacting with impurities and bulk acoustic phonons in the presence of a transverse magnetic field is developed in Ref. 11. The phonons are described by the mode index $\lambda$ and wave vector $\mathbf{Q}=\left(\mathbf{q}, q_{z}\right)$, where $\mathbf{q}$ is the component of the wave vector in the $2 \mathrm{D}$ plane. Under conditions when both cyclotron energy $\hbar \omega_{c}$ and 


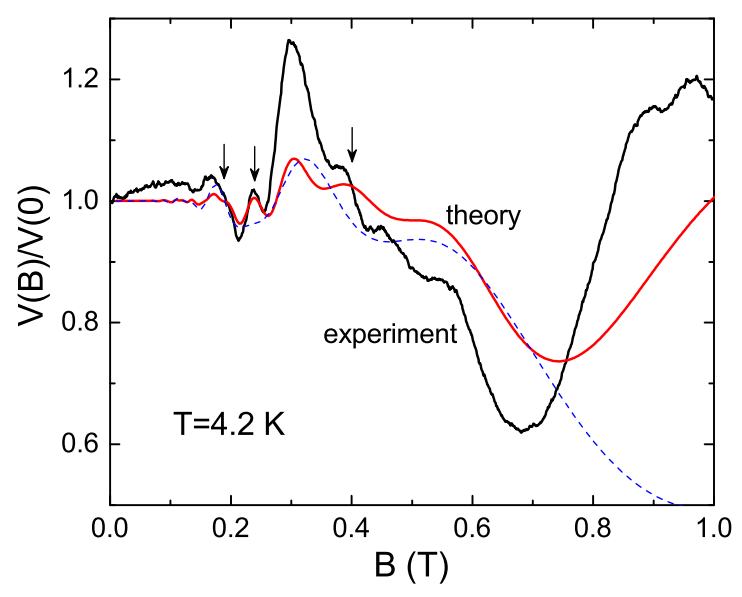

FIG. 5: (Color online) Magnetic-field dependence of the normalized thermoinduced voltage for two-subband QW. The combined resonances are marked by the arrows. The dashed line shows the result of calculation where the intersubband phonon-assisted scattering was intentionally removed.

phonon energy $\hbar \omega_{\lambda \mathbf{Q}}$ are much smaller than the Fermi energy $\varepsilon_{F}$ (the case of degenerate electron gas, $T \ll \varepsilon_{F}$, is assumed), the kinetic equation for the distribution function $f_{j \varepsilon \varphi}$ depending on the subband index $j$, energy $\varepsilon$, and electron momentum angle $\varphi$ is written as

$$
\omega_{c} \frac{\partial f_{j \varepsilon \varphi}}{\partial \varphi}=J_{j \varepsilon \varphi}^{i m}(f)+J_{j \varepsilon \varphi}^{p h}(f)
$$

where $J_{j \varepsilon \varphi}^{i m}$ and $J_{j \varepsilon \varphi}^{p h}$ are the electron-impurity and electron-phonon collision integrals. The expression for $J_{j \varepsilon \varphi}^{p h}$ (Eq. (3) of Ref. 11) needs to be generalized to the case of anisotropic phonon distribution function $N_{\lambda \mathbf{Q}}$ by substituting $N_{\lambda \mathbf{Q}}$ in the phonon emission (first) term and $N_{\lambda-\mathbf{Q}}$ in the phonon absorption (second) term in place of the isotropic Planck distribution $N_{\omega_{\lambda \mathrm{Q}}}$. At temperatures $4.2 \mathrm{~K}$ and lower, the electron-impurity scattering prevails over the electron-phonon one and controls relaxation of electron momentum in the systems with mobilities of the order $10^{6} \mathrm{~cm}^{2} / \mathrm{V} \mathrm{s}$. However, the electron-phonon scattering is the one responsible for the phonon drag effect. The non-equilibrium part of electron distribution, $\delta f_{j \varepsilon \varphi}$, appearing due to the drag effect is found from the equation

$$
\omega_{c} \frac{\partial \delta f_{j \varepsilon \varphi}}{\partial \varphi}=\delta J_{j \varepsilon \varphi}^{p h}\left(f^{(0)}\right)+J_{j \varepsilon \varphi}^{i m}(\delta f)
$$

where $f_{j \varepsilon}^{(0)}$ is the equilibrium Fermi distribution function and $\delta J_{j \varepsilon \varphi}^{p h}$ is the contribution to collision integral caused by the antisymmetric in $\mathbf{Q}$ part of the phonon distribu- tion function, $\delta N_{\lambda \mathbf{Q}}$ :

$$
\begin{aligned}
& \delta J_{j \varepsilon \varphi}^{p h}=\frac{m}{\hbar^{3}} \sum_{j^{\prime}} \int_{0}^{2 \pi} \frac{d \varphi^{\prime}}{2 \pi} \sum_{\lambda} \int_{-\infty}^{\infty} \frac{d q_{z}}{2 \pi} C_{\lambda \mathbf{Q}_{j j^{\prime}}} I_{j j^{\prime}}\left(q_{z}\right) \\
& \times \delta N_{\lambda \mathbf{Q}_{j j^{\prime}}} \sum_{l= \pm 1} l D_{j^{\prime} \varepsilon-l \hbar \omega_{\lambda \mathbf{Q}_{j j^{\prime}}}}\left(f_{j^{\prime} \varepsilon-l \hbar \omega_{\lambda} \mathbf{Q}_{j j^{\prime}}}^{(0)}-f_{j \varepsilon}^{(0)}\right) .
\end{aligned}
$$

The phonon wave vector $\mathbf{Q}_{j j^{\prime}}=\left(\mathbf{q}_{j j^{\prime}}, q_{z}\right)$ in this expression depends on the subband indices. Its in-plane component $\mathbf{q}_{j j^{\prime}}$ is defined by the polar angle $\varphi_{q}=$ $\arctan \left[\left(k_{j} \sin \varphi-k_{j^{\prime}} \sin \varphi^{\prime}\right) /\left(k_{j} \cos \varphi-k_{j^{\prime}} \cos \varphi^{\prime}\right)\right]$ and absolute value $q_{j j^{\prime}}=\sqrt{k_{j}^{2}+k_{j^{\prime}}^{2}-2 k_{j} k_{j^{\prime}} \cos \theta}$, where $k_{j}$ is the Fermi wavenumber in the subband $j$ and $\theta=\varphi-\varphi^{\prime}$ is the scattering angle. Owing to smallness of phonon energies, the quasielastic scattering approximation used in these expressions is justified. Next, $D_{j \varepsilon}$ is the density of states in subband $j$, expressed in units of $m / \pi \hbar^{2}$, $C_{\lambda \mathbf{Q}_{j j^{\prime}}}$ is the squared matrix element of electron-phonon interaction in the bulk, determined by both deformationpotential and piezoelectric mechanisms of the interaction, and

$$
I_{j j^{\prime}}\left(q_{z}\right)=\left|\int d z \Psi_{j}^{*}(z) e^{i q_{z} z} \Psi_{j^{\prime}}(z)\right|^{2}
$$

is the overlap factor determined by the envelope wave functions $\Psi_{j}(z)$ and $\Psi_{j^{\prime}}(z)$ of the corresponding subbands. For a wide quantum well, these factors are to be calculated numerically (Fig. 5).

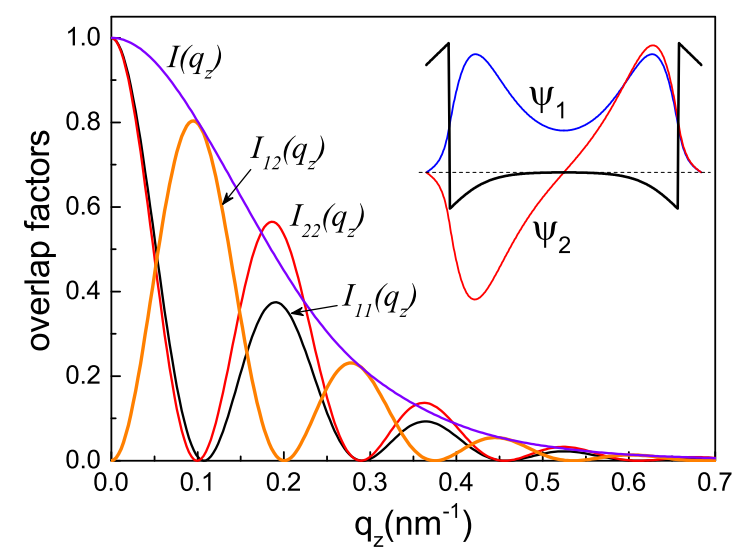

FIG. 6: (Color online) Overlap factors $I_{j j^{\prime}}$ and their combination $I=\left(I_{11}+I_{22}\right) / 2+I_{12}$ for QW of width $w=45 \mathrm{~nm}$ with density $n_{s}=6.4 \times 10^{11} \mathrm{~cm}^{-2}$ studied in our experiment. The inset shows the confinement potential and wave functions of the first (symmetric) and the second (antisymmetric) subband states determined by a self-consistent solution of the Schroedinger and Poisson equations.

Since $J_{j \varepsilon \varphi}^{i m}(\delta f)$ is linear in $\delta f_{j \varepsilon \varphi}$, the kinetic equation (3) with $\delta J_{j \varepsilon \varphi}^{p h}$ of Eq. (4) is solved straightforwardly. The 
thermoelectric current density,

$$
\mathbf{j}_{T}=\frac{e}{\pi \hbar} \sum_{j} \int d \varepsilon D_{j \varepsilon} k_{j} \int_{0}^{2 \pi} \frac{d \varphi}{2 \pi}\left(\begin{array}{c}
\cos \varphi \\
\sin \varphi
\end{array}\right) \delta f_{j \varepsilon \varphi},
$$

is determined by $\delta f_{j \varepsilon \varphi}$. Because of high mobility of electrons, we consider the regime of classically strong magnetic fields, when $\omega_{c}$ is much larger than the inverse transport scattering time $1 / \tau_{t r}$. The zero-order iteration in small parameter $1 / \omega_{c} \tau_{t r}$ is sufficient for calculation of the longitudinal thermopower. The thermoelectric current in this approximation is independent of scattering by impurities:

$$
\begin{array}{r}
\mathbf{j}_{T} \simeq \frac{e}{m \omega_{c}} \sum_{j j^{\prime}}\left(n_{j}+n_{j^{\prime}}\right) \\
\times \hat{\mathcal{G}}_{j j^{\prime}}\left\{q_{j j^{\prime}}^{-1} \delta N_{\lambda \mathbf{Q}_{j j^{\prime}}} R_{j j^{\prime}}\left(\begin{array}{c}
-\sin \varphi_{q} \\
\cos \varphi_{q}
\end{array}\right)\right\},
\end{array}
$$

where $n_{j}=k_{j}^{2} / 2 \pi$ is the electron density in the subband $j, \widehat{\mathcal{G}}_{j j^{\prime}}$ is the integral operator defined as

$$
\begin{aligned}
& \widehat{\mathcal{G}}_{j j^{\prime}}\{A\} \equiv \frac{2 m^{2}}{\hbar^{3}} \int_{0}^{2 \pi} \frac{d \theta}{2 \pi} \int_{0}^{2 \pi} \frac{d \varphi_{q}}{2 \pi} \sum_{\lambda} \int_{-\infty}^{\infty} \frac{d q_{z}}{2 \pi} \\
& \times \omega_{\lambda \mathbf{Q}_{j j^{\prime}}} C_{\lambda \mathbf{Q}_{j j^{\prime}}} I_{j j^{\prime}}\left(q_{z}\right) \mathcal{F}_{j j^{\prime}}(\theta) A, \\
& \mathcal{F}_{j j^{\prime}}(\theta)=1-2 k_{j} k_{j^{\prime}} \cos \theta /\left(k_{j}^{2}+k_{j^{\prime}}^{2}\right) \text {, and } \\
& R_{j j^{\prime}}=\frac{1}{2 \hbar \omega_{\lambda \mathbf{Q}_{j j^{\prime}}}} \int d \varepsilon\left(f_{\varepsilon-\hbar \omega_{\lambda \mathbf{Q}_{j j^{\prime}}}^{(0)}}-f_{\varepsilon}^{(0)}\right) \\
& \times\left(D_{j \varepsilon} D_{j^{\prime} \varepsilon-\hbar \omega_{\lambda \mathbf{Q}_{j j^{\prime}}}}+D_{j^{\prime} \varepsilon} D_{j \varepsilon-\hbar \omega_{\lambda \mathbf{Q}_{j j^{\prime}}}}\right) .
\end{aligned}
$$

Finally, specifying the phonon distribution as [39]

$$
\delta N_{\lambda \mathbf{Q}}=\frac{\partial N_{\omega_{\lambda \mathbf{Q}}}}{\partial \omega_{\lambda \mathbf{Q}}} \frac{\omega_{\lambda \mathbf{Q}}}{T} \tau_{\lambda} \mathbf{u}_{\lambda \mathbf{Q}} \cdot \nabla T,
$$

where $\tau_{\lambda}$ is the phonon lifetime and $\mathbf{u}_{\lambda \mathbf{Q}}=\partial \omega_{\lambda \mathbf{Q}} / \partial \mathbf{Q}$ is the phonon group velocity, one can find the current density in the standard form $\mathbf{j}_{T}=-\hat{\beta} \nabla T$, where the thermoelectric tensor $\hat{\beta}$, in view of the assumed condition $\omega_{c} \tau_{t r} \gg 1$, has only non-diagonal components. The longitudinal thermopower is given by the following expression

$$
\begin{aligned}
& \alpha_{x x} \simeq \rho_{x y} \beta_{y x}=-\frac{1}{\hbar|e|} \sum_{j j^{\prime}} \frac{n_{j}+n_{j^{\prime}}}{2 n_{s}} \\
& \times \hat{\mathcal{G}}_{j j^{\prime}}\left\{\tau_{\lambda} Q_{j j^{\prime}}^{-2} F\left(\frac{\hbar \omega_{\lambda \mathbf{Q}_{j j^{\prime}}}}{2 T}\right) R_{j j^{\prime}}\right\},
\end{aligned}
$$

where $F(x)=[x / \sinh (x)]^{2}$. The Hall resistance is $\rho_{x y}=$ $m \omega_{c} / e^{2} n_{s}$. In the case of single subband occupation, the expression for $\beta_{y x}$ is reduced to the one obtained in Ref. 15. Calculation of the integral over energy in Eq. (9) is considerably simplified under condition $2 \pi^{2} T \gg$ $\hbar \omega$, when Shubnikov-de Haas oscillation are thermally suppressed. At $T=4.2 \mathrm{~K}$ this condition is satisfied up to $B=1 \mathrm{~T}$, so we use it in the following.

Below, for analysis of experimental data, we restrict ourselves by the approximation of overlapping Landau levels, when only the first oscillatory harmonics of the density of states are taken into account: $D_{j \varepsilon} \simeq 1-$ $2 d_{j} \cos \left[2 \pi\left(\varepsilon-\varepsilon_{j}\right) / \hbar \omega_{c}\right]$, where $d_{j}$ are the Dingle factors and $\varepsilon_{j}$ are the quantization energies of the subbands. Since the subband separation $\Delta=\varepsilon_{2}-\varepsilon_{1}$ is much smaller than $2 \varepsilon_{F}$, we also neglect the difference between $k_{1}$ and $k_{2}$. The latter approximation means that $q_{j j^{\prime}} \simeq 2 k_{F} \sin (\theta / 2)$ (so that $\mathbf{Q}_{j j^{\prime}}$ is no longer dependent on the subband indices), $n_{1} \simeq n_{2} \simeq n_{s} / 2$ and $\mathcal{F} \simeq 1-\cos \theta$. Thus, calculation of the sum over the subband indices in Eq. (11) is reduced to calculation of the factor

$$
\begin{aligned}
& \mathcal{I}_{\lambda \mathbf{Q}}\left(q_{z}\right)=\frac{1}{2} \sum_{j j^{\prime}} I_{j j^{\prime}}\left(q_{z}\right) R_{j j^{\prime}} \simeq I\left(q_{z}\right)+\cos \frac{2 \pi \omega_{\lambda \mathbf{Q}}}{\omega_{c}} \\
& \times\left(d_{1}^{2} I_{11}\left(q_{z}\right)+d_{2}^{2} I_{22}\left(q_{z}\right)+2 d_{1} d_{2} I_{12}\left(q_{z}\right) \cos \frac{2 \pi \Delta}{\hbar \omega_{c}}\right),
\end{aligned}
$$

where $I\left(q_{z}\right)=\left(I_{11}+I_{22}\right) / 2+I_{12}$. The thermopower takes the form

$$
\begin{aligned}
& \alpha_{x x} \simeq-\frac{m^{2}}{|e| \hbar^{4}} \int_{0}^{2 \pi} \frac{d \theta}{2 \pi} \int_{0}^{2 \pi} \frac{d \varphi_{q}}{2 \pi} \sum_{\lambda} \int_{0}^{\infty} \frac{d q_{z}}{\pi} \\
& \times(1-\cos \theta) C_{\lambda \mathbf{Q}} \tau_{\lambda} F\left(\frac{\omega_{\lambda \mathbf{Q}}}{2 T}\right) \frac{2 \omega_{\lambda \mathbf{Q}}}{Q^{2}} \mathcal{I}_{\lambda \mathbf{Q}}\left(q_{z}\right) .
\end{aligned}
$$

Comparing Eq. (13) with the results of Ref. 15, one may notice that the single-subband case is described by the substitution $\mathcal{I}_{\lambda \mathbf{Q}} \rightarrow I_{q_{z}}\left[1+2 d^{2} \cos \left(2 \pi \omega_{\lambda \mathbf{Q}} / \omega_{c}\right)\right]$, where $I_{q_{z}}$ is the corresponding overlap factor.

The expression for $\mathcal{I}_{\lambda \mathbf{Q}}\left(q_{z}\right)$ comprises both the classical contribution proportional to $I\left(q_{z}\right)$ and the quantum contribution containing MPO originating from intrasubband (terms at $I_{11}$ and $I_{22}$ ) and intersubband (term at $I_{12}$ ) transitions of electrons. The presence of the product of magnetophonon oscillating factor $\cos \left(2 \pi \omega_{\lambda \mathbf{Q}} / \omega_{c}\right)$ by the magneto-intersubband oscillating factor $\cos \left(2 \pi \Delta / \hbar \omega_{c}\right)$ formally discloses the interference nature of the intersubband term. This product is also representable as a sum of oscillating factors with the combined frequencies $\omega_{\lambda \mathbf{Q}}^{ \pm}=\omega_{\lambda \mathbf{Q}} \pm \Delta / \hbar$ leading to combined resonances according to Eq. (1).

\section{NUMERICAL RESULTS AND DISCUSSION}

The results of numerical calculation of the normalized thermopower $\alpha_{x x}(B) / \alpha_{x x}(0)=V(B) / V(0)$ for both single-subband and two-subband QWs are presented together with the experimental plots in Figs. 3 and 4. The calculation for two-subband $\mathrm{QW}$ are done according to Eqs. (12) and (13), under approximation $d_{1}=d_{2}=$ $\exp \left(-\pi / \omega_{c} \tau\right)$, where $\tau$ is the quantum lifetime of electrons, common for both subbands. 
In the calculations, we used the parameters of our samples together with material parameters of GaAs, substituted $\tau=7$ ps (estimated from the MISO amplitude, see Fig. 1), and assumed $\tau_{\lambda}$ as a mode-independent constant. We need to emphasize that the theory is based on the model form of non-equilibrium part of the phonon distribution function, Eq. (10), while the actual phonon distribution in our experiment is influenced by geometrical details [16] and, therefore, may considerably deviate from this form. For this reason, we do not expect a good agreement between the theory and the experiment as concerns the amplitudes of the oscillations. On the other hand, both the general behavior of the thermoinduced voltage and the position of extrema should be reproduced correctly, and we indeed have this kind of agreement. However, we noticed that the contribution of the transverse phonon modes to the thermoelectric effect is larger than expected from the theory. Since the interaction with transverse modes is associated mostly with the piezoelectric mechanism of electron-phonon interaction, we used the piezoelectric potential constant $h_{14}$ as a single adjustable parameter, to find a better correspondence between the theory and the experiment. We have found that both single-subband and two-subband cases are described considerably better when $h_{14}$ is increased from the usually assumed value of $1.2 \mathrm{~V} / \mathrm{nm}$ to $2.8 \mathrm{~V} / \mathrm{nm}$. The increased importance of transverse modes and/or piezoelectric mechanism in the thermoelectric experiments can be likely associated with excitation of surface acoustic phonon modes as a result of external heating. These phonons interact with 2D electrons mostly via the longrange piezoelectric fields and their frequency is close to the frequencies of the bulk transverse phonons [40]. The problem of the possible contribution of surface acoustic phonons to magnetothermopower oscillations requires a special study which is beyond the scope of the present work.

The characteristic features appearing in the thermoelectric effect in two-subband QWs because of intersubband phonon-assisted scattering deserve a discussion. Since the intersubband separation $\Delta$ in our samples is comparable to resonance phonon frequencies $\omega_{p h}=$ $2 k_{F} s_{\lambda}$, the most prominent manifestation of the combined resonances is the appearance of extra peaks when the MPO minima coincide with MISO minima. In other words, when both $\omega_{p h}$ and $\Delta / \hbar$ are half-integer multi- ples of $\omega_{c}$, the combined frequencies $\omega_{p h} \pm \Delta / \hbar$ are integer multiples of $\omega_{c}$, so instead of a local minimum expected in the absence of intersubband transitions one has a local maximum. We clearly observe this kind of peaks at $B \simeq 0.24 \mathrm{~T}$ and $B \simeq 0.4 \mathrm{~T}$. There are also weaker features such as a barely resolved local minimum at $B \simeq 0.18 \mathrm{~T}$. When the MPO maxima coincide with MISO maxima (both $\omega_{p h}$ and $\Delta / \hbar$ are integer multiples of $\omega_{c}$ ), the enhancement of the thermoinduced voltage takes place, for example, at $B \simeq 0.3 \mathrm{~T}$.

The non-monotonic behavior of the thermoinduced voltage at higher fields is also a consequence of intersubband scattering (compare two theoretical plots in Fig. 4). When $\omega_{c}$ becomes larger than the resonance frequency of the highest-energy phonon mode (in our sample, when $B>0.55 \mathrm{~T}$ ), the probability of phonon-assisted scattering within the same subband decreases monotonically. However, because of enhancement of the phonon-assisted scattering between the adjacent Landau levels of different subbands (these levels are separated by the energy $\left.\hbar \omega_{c}-\Delta\right)$, the thermoinduced voltage passes through a minimum near $B=0.7 \mathrm{~T}$ and then increases.

The intersubband scattering of electrons requires phonons with finite perpendicular component of the wave vector, $q_{z}$. On the other hand, the magnetophonon resonance occurs at $q_{z}$ much smaller than $2 k_{F}$. In wide QWs, where $k_{F} w \gg 1$, these conditions do not contradict with each other, because the overlap factor $I_{12}\left(q_{z}\right)$ becomes sufficiently large already at $q_{z} \ll 2 k_{F}$. The presence of non-equilibrium phonons with finite $q_{z}$ can be explained even in the case of ballistic phonon propagation: such phonons coming from the heater reflect from the upper and lower boundaries of the sample and can reach the $2 \mathrm{D}$ layer.

In conclusion, we observe acoustic magnetophonon oscillations of thermopower in high-mobility 2D electron gas in quantum wells with two occupied $2 \mathrm{D}$ subbands and detect the combined resonances caused by intersubband phonon-assisted transitions of electrons. A detailed comparison of experimental and theoretical dependence of thermopower on magnetic field is carried out. The importance of intersubband transitions in the phonon drag effect in magnetic field is demonstrated.

The financial support of this work by FAPESP, CNPq (Brazilian agencies) is acknowledged.
[1] M. A. Zudov, I.V. Ponomarev, A. L. Efros, R. R. Du, J. A. Simmons, and J. L. Reno, Phys. Rev. Lett. 86, 3614 (2001).

[2] J. Zhang, S. K. Lyo, R. R. Du, J. A. Simmons, and J. L. Reno, Phys. Rev. Lett. 92, 156802 (2004).

[3] A. A. Bykov, A. K. Kalagin and A. K. Bakarov, JETP Lett. 81, 523 (2005).

[4] W. Zhang, M. A. Zudov, L. N. Pfeiffer, and K. W. West, Phys. Rev. Lett. 100, 036805 (2008).
[5] X. L. Lei, Phys. Rev. B 77, 205309 (2008).

[6] A. T. Hatke, M. A. Zudov, L. N. Pfeiffer, and K. W. West, Phys. Rev. Lett. 102, 086808 (2009).

[7] A. A. Bykov and A. V. Goran, JETP Lett., 90, 578 (2009).

[8] O. E. Raichev, Phys. Rev. B 80, 075318 (2009).

[9] A. Bykov, A. Goran, and S. Vitkalov, Phys. Rev. B 81, $155322(2010)$.

[10] O. E. Raichev, Phys. Rev. B 81, 165319 (2010). 
[11] O. E. Raichev, Phys. Rev. B 81, 195301 (2010).

[12] I. A. Dmitriev, R. Gellmann, and M. G. Vavilov, Phys. Rev. B 82, 201311(R) (2010).

[13] A. T. Hatke, M. A. Zudov, L. N. Pfeiffer, and K. W. West, Phys. Rev. B 84, 121301 (2011).

[14] I. A. Dmitriev, A. D. Mirlin, D. G. Polyakov, and M. A. Zudov, Rev. Mod. Phys. 84, 1709 (2012).

[15] O. E. Raichev, Phys. Rev. B 91, 235307 (2015).

[16] A. D. Levin, Z. S. Momtaz, G. M. Gusev, O. E. Raichev, and A. K. Bakarov, Phys. Rev. Lett. 115, 206801 (2015).

[17] For a review of magnetothermoelectric effects, see R. Fletcher, Semicond. Sci. Technol. 14, R1 (1999).

[18] C. Ruf, H. Obloh, B. Junge, E. Gmelin, K. Ploog, and G. Weimann, Phys. Rev. B 37, 6377 (1988).

[19] V. Polyanovsky, Fiz. Tekh. Poluprovodn. 22, 2230 (1988) [Sov. Phys. - Semicond. 22, 1408 (1988)].

[20] P. T. Coleridge, Semicond. Sci. Technol. 5, 961 (1990).

[21] D. R. Leadley, R. Fletcher, R. J. Nicholas, F. Tao, C. T. Foxon, and J. J. Harris, Phys. Rev. B 46, 12439 (1992).

[22] T. H. Sander, S. N. Holmes, J. J. Harris, D. K. Maude, and J. C. Portal, Phys. Rev. B 58, 13856 (1998).

[23] A. C. H. Rowe, J. Nehls, R. A. Stradling, and R.S. Ferguson, Phys. Rev. B 63, 201307(R) (2001).

[24] N. C. Mamani, G. M. Gusev, T. E. Lamas, A. K. Bakarov, and O. E. Raichev, Phys. Rev. B 77, 205327 (2008).

[25] A. A. Bykov, D. P. Islamov, A. V. Goran, A. I. Toropov, JETP Lett. 87, 477 (2008).

[26] M. E. Raikh and T. V. Shahbazyan, Phys. Rev. B 49, 5531 (1994).

[27] N. S. Averkiev, L. E. Golub, S. A. Tarasenko, and M. Willander, J. Phys. - Cond. Matter 13, 2517 (2001).

[28] O. E. Raichev, Phys. Rev. B 78, 125304 (2008).

[29] S. Wiedmann, G. M. Gusev, O. E. Raichev, T. E. Lamas,
A. K. Bakarov, and J. C. Portal, Phys. Rev. B 78, 121301(R) (2008).

[30] N. C. Mamani, G. M. Gusev, O. E. Raichev, T. E. Lamas, and A. K. Bakarov, Phys. Rev. B 80, 075308 (2009).

[31] N. C. Mamani, G. M. Gusev, E. C. F. da Silva, O. E. Raichev, A. A. Quivy, and A. K. Bakarov, Phys. Rev. B 80, 085304 (2009).

[32] S. Wiedmann, N. C. Mamani, G. M. Gusev, O. E. Raichev, A. K. Bakarov, and J. C. Portal, Phys. Rev. B 80, 245306 (2009).

[33] S. Wiedmann G. M. Gusev, O. E. Raichev, A. K. Bakarov, and J. C. Portal, Phys. Rev. B 82, 165333 (2010).

[34] W. Mayer, J. Kanter, J. Shabani, S. Vitkalov, A. K. Bakarov, and A. A. Bykov, Phys. Rev. B 93, 115309 (2016).

[35] Other quantum interference phenomena are observed in nonlinear magnetotransport at strong dc driving and in non-equilibrium magnetotransport under microwave irradiation (see Ref. 14 for a review).

[36] R. J. Hyndman, S. T. Stoddart, B. Tieke, S. G. S. Lok, B. L. Gallagher, A. K. Geim, J. C. Maan, and M. Henini, Physica B 249-251, 745 (1998).

[37] T. Smith, M. Tsaousidou, R. Fletcher, P. T. Coleridge, Z. R. Wasilewski, and Y. Feng, Phys. Rev. B 67, 155328 (2003).

[38] R. Fletcher, T. Smith, M. Tsaousidou, P. T. Coleridge, Z. R. Wasilewski, and Y. Feng Phys. Rev. B 70, 155333 (2004).

[39] A. Miele, R. Fletcher, E. Zaremba, Y. Feng, C. T. Foxon, and J. J. Harris, Phys. Rev. B 58, 13181 (1998).

[40] A. L. Efros and Yu. M. Galperin, Phys. Rev. Lett. 64, 1959 (1990) and references therein. 